点 $A_{i}^{(n)}, A_{i}^{(n)}$,

$$
d_{\mathscr{X}^{n}(G)}\left(A_{1}^{(n)}, A_{2}^{(n)}\right)=\operatorname{diam} G+n
$$

当且仅当对任何

$$
u \in \mathscr{B}_{0}\left(A_{1}^{(n)}\right), v \in \mathscr{B}_{0}\left(A_{2}^{(n)}\right)
$$

均有 $d_{G}(u, v)=\operatorname{diam} G$. 其中 $\mathscr{B}_{0}\left(A^{(n)}\right)$ 表示 $\mathscr{K}^{\prime \prime}(G)$ 的节点 $A^{(s)}$ 相应的在 $G$ 中 的 节点子集.

对于二图 $G_{1}=\left(V_{1}, E_{1}\right), G_{2}=\left(V_{2}\right.$, $\left.E_{2}\right),\left|V_{1}\right|=\left|V_{2}\right|=m$. 记

$$
V_{1}=\left\{u_{1}, u_{2}, \cdots, u_{m}\right\},
$$

$V_{2}=\left\{v_{1}, v_{2}, \cdots, v_{m}\right\}$.

$Q\left(G_{1}, G_{2}\right)=\left(V_{1}+V\left(K_{2 m}\right)+V_{2}, E_{1}\right.$

$$
\left.+L\left(G_{1}\right)+E\left(K_{2 m}\right)+L\left(G_{2}\right)+E_{2}\right),
$$

其中 $K_{2 m}$ 为 $2 m$ 阶的完全图和记

$$
\begin{gathered}
V\left(K_{2 m}\right)=\left\{a_{1}, b_{1}, a_{2}, b_{2}, \cdots \cdots, a_{m}, b_{m}\right\}, \\
L\left(G_{1}\right)=\left\{\left(u_{i}, a_{i}\right) \mid 1 \leqslant i \leqslant m\right\}, \\
L\left(G_{2}\right)=\left\{\left(v_{i}, b_{i}\right) \mid 1 \leqslant i \leqslant m\right\} .
\end{gathered}
$$

定理 2 若令 $\mathscr{E}^{*}(G)$ 表示图 $G$ 的 $n$ 次边图,

$$
H_{n}=Q\left(G_{1}, G_{2}\right), G_{1} \cong G_{2} \cong \mathscr{E}^{n}\left(K_{m}\right) .
$$

则, 对于任何整数 $n \geqslant 0, m \geqslant n+3$, 均有 $\operatorname{diam} \mathscr{K}^{n+1}\left(H_{n}\right)=\operatorname{diam} H_{n}+(n+1)$.

由此可见,定理 2 直接导 致 PRS-猜想 的完满解决.

若在一个图 $G=(V, E)$ 上,对于任何 $x \in V$, 记 $\mathscr{C}_{x}=\left\{c_{x} \mid c_{x}\right.$ 为 $G$ 中含 $x$ 的团 $\}$, 均不存在 $c \bar{E} \mathscr{C}_{x}$ 使得 $c \cap c_{x} \neq \phi, c_{x} \in \mathscr{C}_{x}$ 且对于任何 $(x, y) \in E$ 均有

$$
N_{x}-\{y\} \neq N_{y}-\{x\},
$$

则称 $G$ 具有性质 $\boldsymbol{Q}$. 进而, 还有

定理 3 若 $K_{m} \subseteq G$ 且 $G$ 具有性质 $\Omega$, 则对于任何整数 $n \geqslant 0, m \geqslant n+3$, 均有 $\operatorname{diam} \mathscr{K}^{n}\left(F_{n}\right)=\operatorname{diam} F_{n}+(n+1)$,

其中 $F_{n}=Q\left(\mathscr{E}^{n}(G), \mathscr{E}^{n}(G)\right)$.

然而, 如何刻划方程 (1) 的解集尚有待 进一步解决.

\section{刘彦佩 董峰明}

(中国科学院应用数学研究所, 北京)

李样贵

（云南民族学院数学教研空,昆明）

\title{
交错群的一个新刻划
}

T. Oyama 曾证明如下结论: 若有限群 $G$ 与交错群 $A_{*}$ 有相同的特征表, 则

$$
G \simeq A_{n} \text {. }
$$

本文推广上述结论, 证明了如下定理。

定理 设 $G$ 是群, $\pi_{e}(G)$ 为 $G$ 中元的 阶之集. 则 $G \simeq A_{n}$ 的充要条件是:

(1) $\pi_{e}(G)=\pi_{e}\left(A_{n}\right)$,

(2) $|G|=\left|A_{n}\right|$.

对于某些交错群, 如 $A_{5}, A_{7}, A_{8}$ 仅用定 理中的条件 (1) 即可给出刻划.

为了证明上述定理,我们引人数论函数:

$$
t_{n}(1)=\prod_{\frac{n}{2}<p_{1}<n} p_{1} \text {, }
$$

$$
\begin{aligned}
& t_{n}(2)=\prod_{\frac{n}{3}<p_{2}<i} p_{2}^{2} \cdot t_{n}(1), \cdots, \\
& t_{n}(k)=\prod_{i=1}^{k}\left(\prod_{\frac{n}{i+1}<p_{i}<\frac{n}{i}} p_{i}\right)^{i},
\end{aligned}
$$

其中当 $\frac{n}{j+1}$ 和 $\frac{n}{j}$ 之间没有素数时, $p_{i}$ 定 义为 1 .

令 $G_{n}$ 为满足条件 $\pi_{c}\left(G_{n}\right)=\pi_{c}\left(A_{n}\right)$ 和 $\left|G_{n}\right|=\left|A_{n}\right|$ 的群,我们有下述引理.

引理 $G_{n}$ 是不可解群, $n \geqslant 5$. 若 $n \geqslant 11$, 则 $G_{n}$ 有正规列

$$
G_{n} \geqslant H>N \geqslant 1 \text {, }
$$


其中 $H / N$ 为非交换单群, 且 $t_{\text {* }}(1)|| H / N \mid$.

施武杰

若 $n \geqslant 46$, 则 $t_{n}(3)|| H / N \mid$. 若 $n \geqslant 83$, 则 $t_{n}(6)|| H / N \mid$.

（西南师范大学数学系, 重庆)

毕建行

此引理对定理的证明起着关键的作用.

(辽宁大学数学系,沈阳).

\section{关于二部竞赛图的 Alspach 问题*}

定向图是指无环、无重弧、无 2-有向回 路的有向图. 设 $D=(V, A)$ 是一个 $p$ 阶 定向图, $V$ 和 $A$ 分别表示 $D$ 的点集和 弧 集. 令 $2 \leqslant k \leqslant p-1$ 为整数, 定义

$$
D \in P_{k}\left(D \in P_{k}^{\prime}\right)
$$

当且仅当 $\forall u v \in A(D)$, 在 $D$ 中存在长度 为 $k$ 的 $v-u$ 有向路 ( $u-v$ 有向路). 下记 (i)

$$
D \in \bigcap_{k=1}^{i} P_{k}
$$

和 $D \in \bigcap_{k=i}^{\prime} P_{k}^{\prime}$ 分别表示 $D \in P_{i}$,

$$
D \in P_{i+1} \cdots D \in P_{i}
$$

和 $D \in P_{i}^{\prime}, D \in P_{t+1}^{\prime} \cdots D \in P_{i}^{\prime}$. (ii) $d^{+}(v)$ 和 $d^{-}(v)$ 分别表示顶点 $v$ 在 $D$ 中的出 度和人 度. 如果 $\forall v \in V(D), d^{+}(v)=d^{-}(v)=r$ ( $r \geqslant 1$ 为整数), 则称 $D$ 是 $r$ 正则的.

竞赛图和二部竞赛图是两类特殊的定向 图,其基础图分别为完全图和二部 完 全图. 1967 年 Alspach ${ }^{[1]}$ 证明了: p 阶正则竟赛 图 $T \in \bigcap_{k=2}^{p-1} P_{k} .1974$ 年, Alspach, Reid 和 Roselle $^{[2]}$ 进一步证明了: $p(\geqslant 7)$ 阶正则竟
赛图 $T \in \bigcap_{k=3}^{p-1} P_{k}^{\prime}$. 对于正则二部竞赛图相应 的 Alspach 结果, 1987 年我们在文献[3]中 证明了: $p$ 阶正则二部竞赛图

$$
R \in \bigcap_{k=2}^{p / 2} P_{2 k-1}
$$

除非 $R \cong R_{p}^{*}$. 其中 $R_{\text {* }}^{*}$ 是一个特殊 的二 部竞赛图, $V\left(R_{4 r}^{*}\right)=\left\{v_{1}, v_{2} \cdots v_{4 r} . r \geqslant 1\right.$ 为整数\},

$$
A\left(R_{4}^{*}\right)=\left\{v_{i} v_{i} \mid j-i \equiv 1(\bmod 4)\right\},
$$

在本文中,我们得到了下面的结果.

定理 $p$ 阶正则二部竞赛图

除非 $R \cong R_{p}^{*}$.

$$
R \in \bigcap_{k=2}^{p / 2} P_{2 k-1}^{\prime},
$$

\section{参考文 献}

[1] Alspach, B., Canad. Math. Bull., 10(1967). $283-286$.

[2] Alspach, B. et al., J. C. T., B17 (1974), 1118.

[3]王建中、何曙光,科学通报, 32 (1987), 1:76.

王建中 (太原机械学院理学系)

\footnotetext{
- 太原机械学院科研基金资助课题.
}

\section{集合搜索作为具有布尔代数元的 IF-THEN 结构}

文献 [1] 从问题求解的角度提出, 集合 搜索同构于一个布尔代数, 即:

$$
\text { 命题 } 1 \quad(s, \stackrel{*}{\rightarrow}) \simeq\left(2^{v}, U, \cap, C\right)
$$

作者在近期研究中发现, 集合搜索实质 上是具有布尔代数 元 的 IF-THEN 结构, 即:

\author{
命题 $2(S, \stackrel{*}{\longrightarrow}) \simeq\left(\right.$ IF $X_{I}$ THEN \\ $\left.Y_{U}\right)$
}

在这儿, $X_{I}$ 和 $Y_{U}$ 分别为一个相应的布尔代 数,其中,下标 $I$ 是启发信息* 的全部信息空 间或信息全域，而下标 $U$ 是搜索过程 $\rightarrow$ 所相: 应的全部状态空间 $S$ 、或搜索全域 $U, X_{I}$ 和. 